\title{
Validity, Reliability and Equivalence of Parallel Examinations in a University Setting*
}

\author{
Bunmi S. Malau-Aduli, Justin Walls, Craig Zimitat \\ School of Medicine, University of Tasmania, Hobart, Australia \\ Email: bunmi.malauaduli@utas.edu.au, justin.walls@utas.edu.au, craig.zimitat@utas.edu.au
}

Received July $9^{\text {th }}$, 2012; revised August $10^{\text {th }}$, 2012; accepted August 22 $2^{\text {nd }}, 2012$

\begin{abstract}
A key issue to address in the design and implementation of any assessment system is ensuring its reliability and validity. University assessment policies often require staff to prepare parallel examinations for students who are unable to sit the initial examination. There is little published literature to give confidence to staff or students that these examinations are indeed reliable or equivalent. This study was conducted to determine the validity, reliability and equivalence of two parallel examinations that have been developed under highly defined quality assurance (QA) processes in a university setting. Collated assessment results for all the 76 participants who sat the parallel examinations were subjected to statistical and correlational analysis to test for significant differences between mean scores and their associated standard deviations. Item analysis was conducted for each assessment by computing the difficulty index (DIF), discrimination index (DI) and Kuder-Richardson 20 (KR-20) reliability using classical test theory. Results indicated comparative proportions of difficulty, functional distractors and internal consistency of the assessment items on both examinations. Comparison of student performances in both examinations revealed that there was no significant difference in mean scores. However, a highly positive and significant correlation $(r=0.82)$ between student total scores in both examinations was evident. Approximately two thirds (62.5 $\%)$ of students with low scores in the first examination also achieved low scores in the second examination. Furthermore, two thirds of the students were ranked in the same order based on performance in both examinations. The established QA processes for assessment in the school provided a strong basis for the generation of multiple sources of data to support arguments for the validity of examinations. It is possible to develop valid, reliable and equivalent parallel tests in university settings with the presence of well-defined QA processes.
\end{abstract}

Keywords: Parallel Examinations; Quality Assurance; Assessment

\section{Introduction}

Universities place considerable emphasis on the development of policies and guidelines that govern examination processes. Well documented policies, strategies and processes, blueprinting to facilitate adequate sampling, feedback to students and assessors and evaluation of the overall process are important elements of any assessment enterprise, however Fowell et al. (1999) argue that insufficient attention is paid to the evaluation of assessment. The latter can be usefully elaborated to include psychometric or statistical analysis of components of the examination or items, establishment of measures of reliability and benchmarking to review pass/fail standards for the examination. Kane (2006) and Schuwirth et al. (2011) identify key questions in support of validity arguments, and highlight the importance of validity data in supporting consistent decision making as a result of assessment. The collation of evaluation data is necessary to provide supporting evidence, and hence confidence in the inferences that will be drawn from assessment in higher education (Kane, 2006). In this case study, we demonstrate the value of quality assurance processes in the generation of evidence to support validity of assessment activities in a medical course.

In Australian universities, assessment policies hold to tenets

"Declaration of Interest: The authors report no conflicts of interest. of reliability, validity and fairness. Fairness includes notions of reliability, validity, transparency and ethical decision making, but it also means that students should be given equal opportunity to demonstrate their learning, free of any disadvantage through identification, language, disability or illness. In the latter case, assessors prepare two or three equivalent versions of each examination (i.e. parallel forms of the test (Tavakol \& Dennick, 2011) - the ordinary examination (OE) that most students sit, a deferred ordinary examination (DO) for students who were ill or unable to sit the ordinary examination, and a supplementary (Supp) examination for students with borderline scores. (The names vary across Australian medical courses). It is assumed that each of these examinations is valid, reliable and equivalent (Norcini et al., 2011), although there is usually insufficient psychometric or statistical data to support such assumptions. This paper aims to provide evidence of the validity, reliability and equivalence of such parallel examinations/tests.

Contemporary assessment theory considers the primacy of construct validity, which draws upon theory and evidence to give meaning to assessment. Typically evidence for validity is drawn from five areas to support confidence in the inferences made from assessment: curriculum content; data management; statistical analyses of test data; correlational analyses; and effects of assessment (Kane, 2006; Downing \& Haladyna, 2009). These are not mutually exclusive evidence categories. The spe- 
cific mix of evidence needed for validation depends on the inferences being drawn and the assumptions being made (Messick, 1989), and extends beyond the validity of the assessment instruments that generate test score data. As assessment regimes become more complex and the stakes related to assessment outcomes increase, the greater the need for multiple sources of data to support the validity of assessment. Our assessment quality assurance (QA) processes were conceptualised in line with current unitary validity theory (Kane, 2006; Downing \& Haladyna, 2009) to generate evidence for medium stakes examinations. The requirement for validity evidence for assessment through the early years of our medical course is modest; however it peaks at the preclinical-clinical transition and graduating examinations.

Academic staff is typically focused on writing questions from their discipline, usually at the expense of the "bigger picture" and question quality. Curriculum content evidence for validity relates to the selection of assessment instruments, alignment of assessment tasks/items with intended learning outcomes, sampling of items across domains of the curriculum, examiner training and the quality of test items. Blueprinting serves to guide the selection of specific assessment instruments, strategies, and more importantly, their development through the specification of the content to be assessed (Hamdy, 2006). Blueprinting mitigates against two significant threats to validity, "construct under-representation" (CU), the biased or undersampling of course content and "construct irrelevance” (CI). CI may arise from a "systematic" error as a result of the poor choice of assessment instrument for the outcomes being assessed (Downing, 2002). But it may also affect a small proportion of students if it arises from poor training of assessors and role player/standardised patients such that students are not exposed to the same test stimuli (e.g. at different sites) or assessed in the same manner. In this case study, each of the examinations were blueprinted to ensure representative and consistent sampling across content domains and alignment with learning outcomes (Jozefowicz et al., 2002; Hamdy, 2006; Hays, 2008), assessors were trained, and internal peer review processes (Malau-Aduli \& Zimitat, 2011) were used to evaluate coverage and to refine test items.

Ensuring the integrity of data arising from the administration of assessment is key for any validity argument. In the first instance, identity and fraud management tag assessment data to the correct individual. The use of double data entry methods and optical scanning forms with checking by software algorithms improves the accuracy of data transfers. Software was developed to automate some processes, particularly those related to generating psychometric reports and student feedback increasing efficiency and decreasing chances for human error to affect data management. The introduction of quality assurance processes, assessment training manuals and automated reporting have all provided significant improvement in consistency of data handling and greater confidence in systems.

Statistical and correlational analyses of assessment data provide important evidence to support or refute validity claims. Item analyses-difficulty, discrimination and internal consistency of the test, inter-rater reliability etc.-are routinely performed as part of quality assurance processes to provide indices of reliability (Tavakol \& Dennick, 2011). Reliability refers to the stability of test scores upon re-testing of examinees which is a fundamental requirement for making meaningful interpretations of test data. Correlational validity evidence may be used to assert positive relationships between performance on two tests of similar abilities (e.g. as students progress through the course), or conversely negative correlations between scores on tests of different abilities. High stakes examinations have high reliability thresholds, whereas it is moderate for many medical course examinations except the final clinical examinations. In our case study, statistical and correlational validity evidence are routinely generated by the QA process.

The final type of data for validity evidence is drawn from the decision making process and its consequences for examinees and others. The documentation of standard setting processes, standard error of measurement associated with cut scores and use of coded candidate details all contribute to this evidence. However it can also extend to correlation of assessment outcomes with later assessments (specialty examinations) or elements of professional practice. Benchmarking of assessment processes and graduate outcomes are more recent types of evidence collected to support assertions of course quality as well as assessment. Some of this data can be collected immediately, and is part of our quality assurance processes, whereas data relating to postgraduate activity falls within the realm of course evaluation.

\section{Context of the Case Study}

The Tasmanian School of Medicine (TSoM) offers a fiveyear case-based undergraduate medical degree. Vertical integration of the curriculum is promoted through a thematic structure usage in all the five years of the program. The first two years of the course provide a systems-based introduction to the foundations of medicine, with an early opportunity to develop communication and clinical skills. Assessment involves formative and summative elements, with an emphasis on end of semester examinations. Well-developed QA processes around assessment (Malau-Aduli et al., 2011) were implemented at the TSoM in 2009 by the Medical Education Unit (MEU). These processes included blueprinting of educational objectives, selecting appropriate test formats and applying assessment strategies to achieve adequate levels of reliability. They also included the implementation of appropriate standard-setting, assessor and role-player training, decision-making procedures and peer review of assessment items to minimise item writing flaws prior to being administered to students (Malau-Aduli et al. 2011).

This case study refers to an examination at the end of the second year of the medical course. QA processes were followed and the three parallel written examination papers (OE, DO and Supp) were developed by faculty at the same time. The university central Examination Office set up new processes to facilitate automated printing of examination papers at the same time as the TSoM established the new QA processes around assessment. The independent and simultaneous introduction of the two new systems in the TSoM and the Examination Office resulted in the automated printing of the examination papers (OE) with answers on them. The examination was re-administered to all students using the DO paper. Students were also offered the opportunity to "resit" the examination in 3 days, using the Supp paper. On the basis of fairness, their result was based upon the highest score achieved on either examination. This rare occasion of administering a "repeat" examination to the same cohort of students provided an ideal opportunity to evaluate the School's assessment practice, using parallel forms 
reliability estimates. Therefore, the objective of this paper is to evaluate the validity, reliability and equivalence of these parallel tests that were developed under well-defined quality assurance (QA) processes.

\section{Methods}

\section{Participants and Procedures}

Second year medical students $(\mathrm{N}=114)$ sitting an integrated basic and clinical science (OE) examination were inadvertently administered a MCQ examination paper (OE) which included answers. The examination was re-administered to all students, using the parallel (DO) paper. However, only seventy-six (76) out of the one hundred and fourteen (114) students in the cohort sat the equivalent examination (Supp) three days later. The scores of these 76 students from the dataset were used for evaluation of these examinations. Clearance was obtained from the relevant ethics committee for this study.

\section{Data Analysis}

Collated assessment results for all the 76 participants who sat both examinations were subjected to statistical analysis using general linear model (GLM) procedure in SAS (SAS, 2009) in a multivariate least squares analysis of variance to test for significant differences between mean scores, their associated standard deviations and descriptive statistics of all the variables. Student scores were compared in the parallel examinations. Significance at the $5 \%$ level was established using the least significant difference technique, while Duncan's multiple range tests was used for mean separation where significant differences were detected. Item analysis was conducted for each assessment by computing the difficulty index (DIF), discrimination index (DI) and Kuder-Richardson 20 (KR-20) reliability using the classical test theory as provided in IDEAL 4.1, an Item Analysis Program (Precht et al., 2003). Details of the quality criteria chosen for each of these quality indicators have been described previously (Malau-Aduli \& Zimitat, 2011). Means of item difficulty, discrimination index and number of functioning distracters per item for all the MCQs were also computed.

\section{Results}

\section{Comparative Analysis of the Two Examinations}

Descriptive statistics for the MCQ examinations are portrayed in Table 1. A comparative appraisal of student performances in both examinations revealed no significant difference in mean scores (Table 1). However, there was a highly significant $(p<0.001)$ and positive correlation $(r=0.82)$ between total student scores in both examinations (not shown). Although the differences were not statistically significant, absolute mean scores were observed to be higher in the first examination than in the second examination (36.4 vs. 33.8). However, there were higher minimum and maximum scores in the second examination (19.0 vs. 14.0 and 46.0 vs. 45.0 , respectively). The first examination recorded a higher reliability index compared to the second examination ( 0.71 vs. 0.68$)$. Similar trends were observed in student performances in the different disciplines assessed in both examinations.

Significantly higher $(\mathrm{p}<0.01)$ mean scores were achieved in Pathology and Pharmacology (75.34\% vs. $75.58 \%$; $69.91 \%$ vs.
Table 1.

Descriptive statistics for both examinations.

\begin{tabular}{ccc}
\hline Criteria & First Exam & Second Exam \\
\hline Number of Items in Exam & 50 & 50 \\
Number of Examinees & 76 & 76 \\
Mean Score & 36.4 & 33.2 \\
Minimum Score & 14.0 & 19.0 \\
Maximum Score & 45.0 & 46.0 \\
Standard Deviation & 5.3 & 4.8 \\
Reliability Index & 0.71 & 0.68 \\
\hline
\end{tabular}

$70.88 \%$ respectively) compared to the other assessed disciplines in both examinations (Figure 1). Students performed somewhat better in the first set of Biochemistry questions than the second and vice versa in the Gross Anatomy questions.

In accordance with the University regulations, the pass mark for each examination was set at $50 \%$. Individual student performances revealed that one student failed both examinations with $30.27 \%$ in the first examination and $37.57 \%$ in the second examination. Approximately two thirds (62\%) of the students with low scores in the first examination also achieved low scores in the second examination. Figure 2 shows the linear regression of student scores in the parallel examinations. The coefficient of determination $\left(\mathrm{R}^{2}=0.67\right)$ is indicative of the precision accuracy that explains $67 \%$ of the observed variation between students' scores in both examinations. Two thirds of the students were ranked in the same order based on performance in both examinations. Compared with performance on the first examination, $5 \%$ of students ranked higher on the second examination, while $30 \%$ ranked lower in the second exam (Figure 3).

\section{Item Analysis}

Table 2 shows the item analysis results for both examinations. Mean difficulty of the test items on the two examinations was similar ( $74 \%$ vs. $67 \%$ ). On the first examination, twentyfour items lay outside the reference range for difficulty level (DIF): all the 24 items appeared to be too easy. Seventeen items on this examination showed very low discrimination $(0-0.15)$ between students who achieved scores in the highest and lowest quartiles. Sixty six percent $(n=33)$ of the items had significantly high discrimination indices with a mean discrimination index of 0.26 . One third of the items on this examination had distractors that were not functioning effectively in their role. The reliability coefficient (KR-20), which is a measure of the internal consistency of the test, was 0.71 .

On the second examination, seventeen items lay outside the reference range for DIF; sixteen of the items appeared to be too easy, whilst one appeared too difficult. Thirteen items on this examination showed very low discrimination between students who achieved scores in the highest and lowest quartiles. Seventy percent $(n=35)$ of the items had high discrimination indices with a mean discrimination index of 0.21 . One third of the items on this examination had distractors which were not functioning effectively in their role. The reliability coefficient (KR-20) was 0.68 .

Distractor analyses were completed for the test items on both 


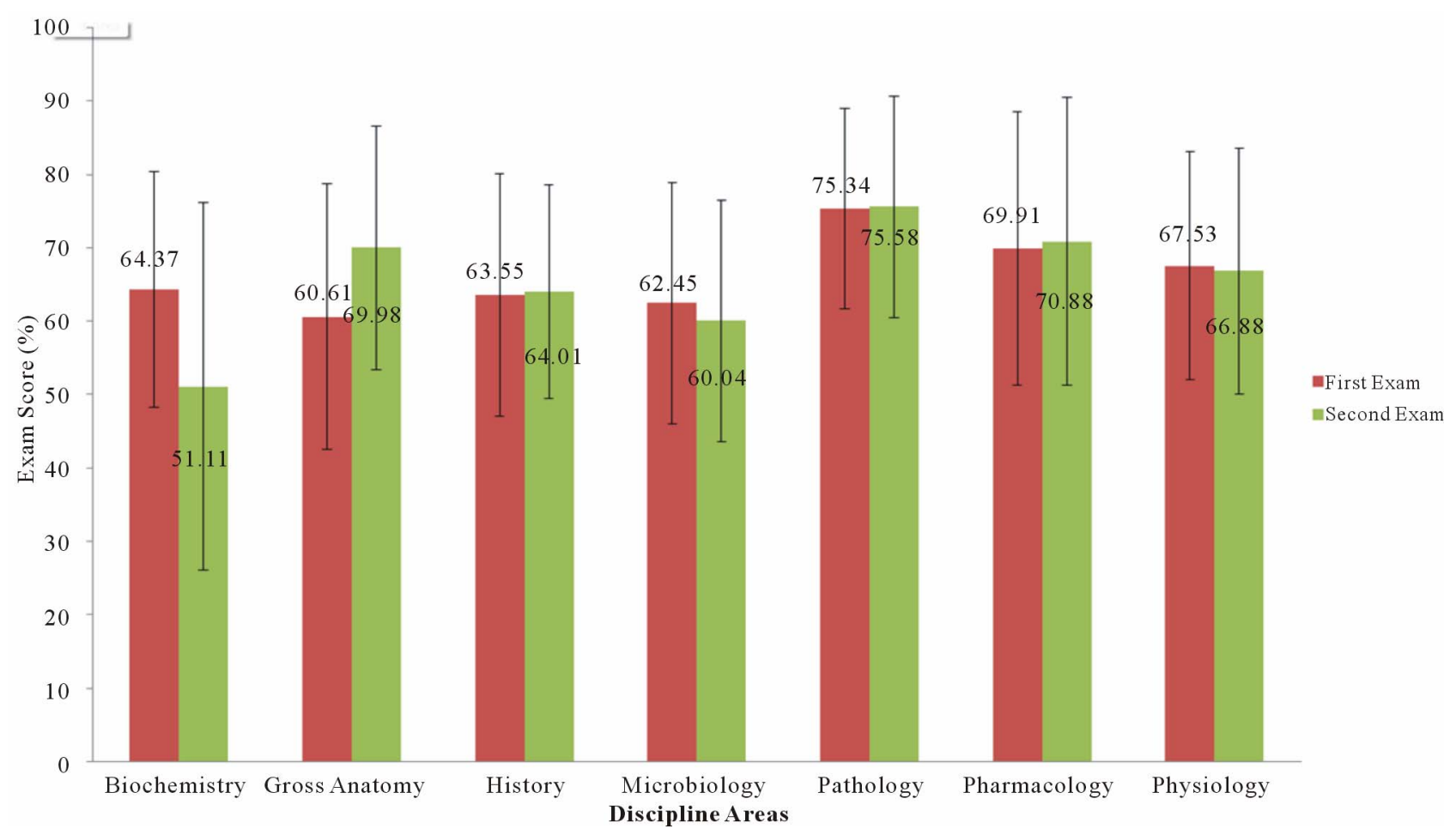

Figure 1.

Comparison of students' performances in both examinations across the different disciplines.

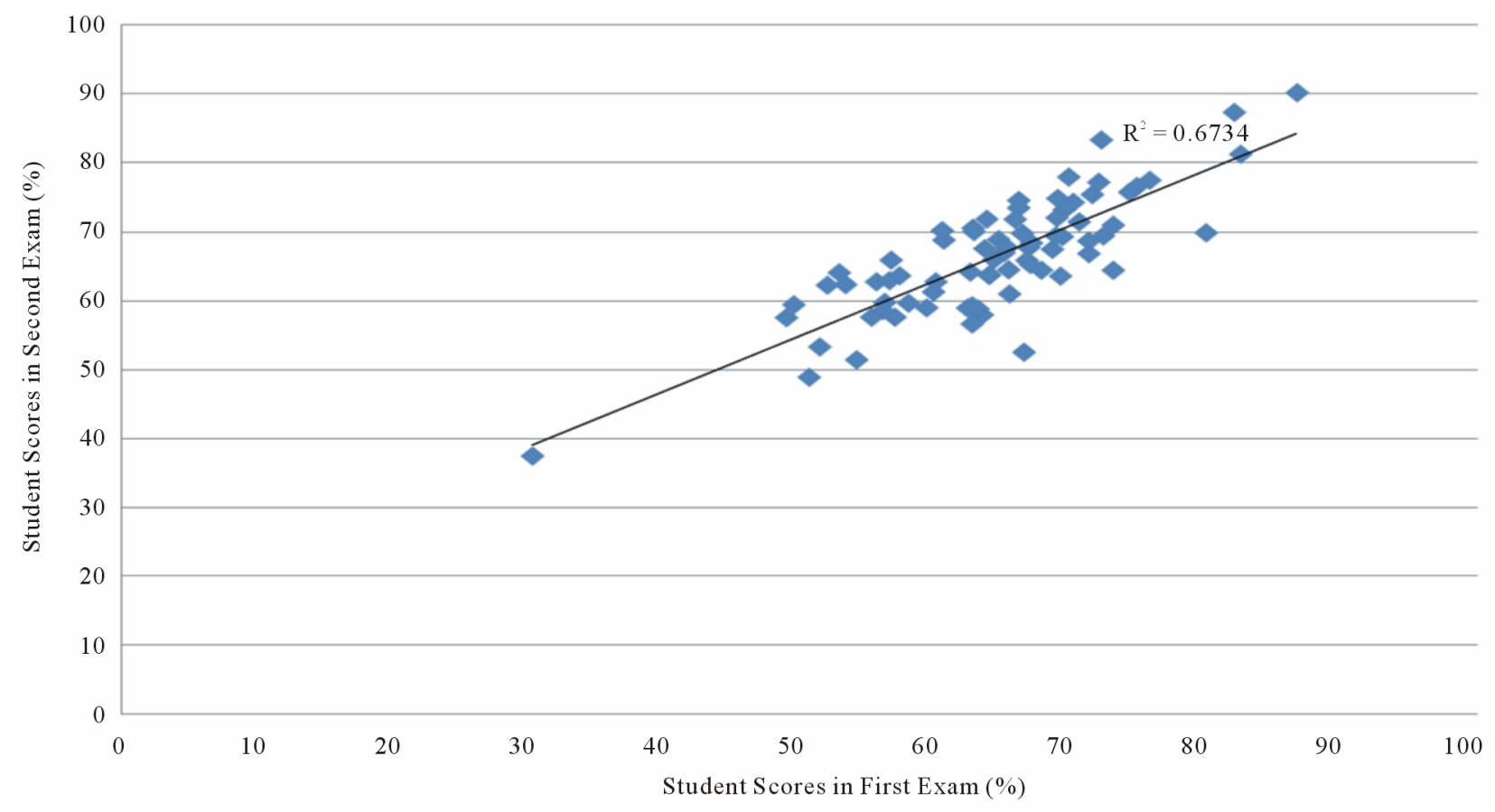

Figure 2.

Comparison of students' performances in both examinations across the different disciplines.

examinations to identify non-functional distractors within each MCQ item. Two hundred distractors associated with the fifty (50) MCQs were assessed in each of the two examinations. Similar patterns were observed in both examinations. In the first examination, $66 \%(n=132)$ of the distractors were func- tional in comparison to $65 \%(\mathrm{n}=130)$ in the second examination. In the first examination, $9.5 \%$ of the distractors were not chosen by any examinee (i.e. the answer key was obvious) compared to $10.5 \%$ in the second examination (Table 3). The mean number of functioning distractors per item was 2.64 in 


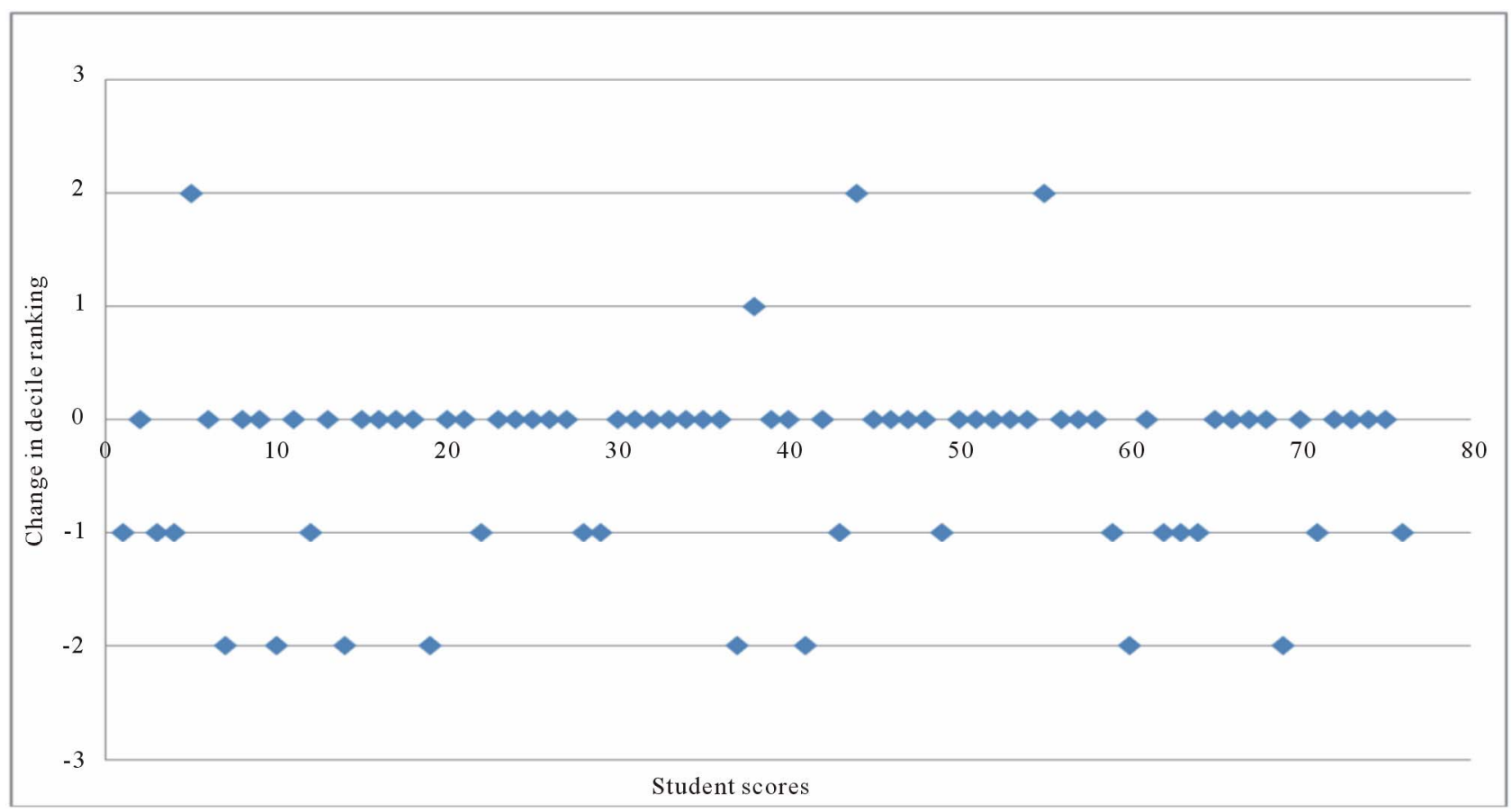

Figure 3.

Change in decile ranking of students in both examinations.

Table 2.

Item analysis for both examinations.

\begin{tabular}{ccc}
\hline Criteria & First Exam & Second Exam \\
\hline Number of Items in Examination & 50.00 & 50.00 \\
Number of Examinees & 76 & 76 \\
Mean Difficulty \% (SD) & $74.28(18.05)$ & $66.46(20.77)$ \\
Mean Discrimination Index (SD) & $0.26(0.15)$ & $0.21(0.13)$ \\
Reliability Index & 0.71 & 0.68 \\
Easy Items (\%) & $24(48)$ & $16(32)$ \\
Difficult Items (\%) & $0(0)$ & $1(2)$ \\
Items with Negative & $0(0)$ & $0(0)$ \\
Discrimination Indices (\%) \\
$\begin{array}{c}\text { Items with Zero } \\
\text { Discrimination Indices (\%) } \\
\text { Items with Low } \\
\text { Discrimination indices (\%) } \\
\text { Total No of Discriminating } \\
\text { Items (\%) }\end{array}$ & $0(0)$ & $2(4)$ \\
\hline
\end{tabular}

the first examination and 2.60 in the second examination. There was a similar pattern in the distribution of functioning distractors per item in both examinations, with an increase in the percentage of items with two and three functional distractors (28\% \& $16 \%$ for the first examination; $34 \%$ \& $12 \%$ for the second examination. In the first examination, there were $28 \%$ of items with one functional distractor and $8 \%$ of items with four functional distractors vs. $24 \%$ and $4 \%$ respectively in the second examination.
Table 3.

Distractor analysis for both examinations.

\begin{tabular}{ccc}
\hline Criteria & First Exam & Second Exam \\
\hline Number of Items in examination & 50 & 50 \\
No of distractors assessed & 200 & 200 \\
Distractors with frequency = 0\% n (\%) & $19(9.5)$ & $21(10.5)$ \\
Distractors with frequency < 5\% n (\%) & $49(24.5)$ & $49(24.5)$ \\
Functioning distractors per test n (\%) & $132(66)$ & $130(65)$ \\
Functioning distractors per item M (SD) & $2.64(1.21)$ & $2.6(1.14)$ \\
Functioning distractors per item n (\%) & \\
None & $10(20)$ & $6(12)$ \\
One & $14(28)$ & $12(24)$ \\
Two & $14(28)$ & $17(34)$ \\
Three & $8(16)$ & $6(12)$ \\
Four & $4(8)$ & $2(4)$ \\
\hline
\end{tabular}

\section{Discussion}

This case study illustrates the value of QA processes in the generation of validity evidence for parallel forms tests typically used in university assessment. These processes generated qualitative and quantitative data in the areas of curriculum content, data management, and statistical and correlational analyses in the face of a major incident affecting our examinations. Since the QA processes were applied in the development of all the test items, we have drawn upon data generated through QA processes to test that assertion and the validity of the two parallel examinations (DO, Supp). 
The QA processes provided qualitative evidence to support argument for curriculum content validity. Developing, blueprinting and editing all the test items for both examinations at the same time ensured that the test items on both examinations covered similar content domains and were aligned to the learning outcomes underscoring the reports by Jozefowicz et al. (2002), Hamdy (2006), Malau-Aduli \& Zimitat (2011). This is indicated in the similar overall mean scores and disciplines/ subcategories scores obtained by students in both examinations. The comparative disciplines/subcategories results also echo equal levels of quality and difficulty of the questions in both examinations. The high correlation between the mean scores on both examinations $(r=0.82)$ indicates that the sets of test items measured the same content area/construct. Results from this study show the comparative proportions of easy, difficult, recall, non-discriminating and non-functional items in both examinations. The observed difference in scores in Biochemistry and Gross Anatomy may be a reflection of how students studied for the second examination (Supp), after the experience of sitting the first examination (DO). In this case, routine statistical data arising from QA processes also support the content equivalence of the two examinations. Data management for the two examinations was undertaken according to the QA process with mechanisms such as key validation, double entry and accuracy of scores.

Statistical and correlational analyses of examination data are routinely conducted to provide evidence of reliability as part of QA processes. The observed high reliability indices and similar student ranking in both examinations indicate that with the second examination, examinees obtained similar scores on retesting (Supp examination) as they did on the first (DO examination). The summary statistics (item analysis) indicated similar trends in performance prompts, discrimination indices, and functionality of distractors and internal consistency reliability of both examinations. Reliability coefficients allow the quantification and estimation of the random errors of measurement in assessment (Downing, 2004). The resulting high prediction accuracy (67\%), and correlation coefficient (0.82) in the comparison of both examinations in this study is an indication of the convergence of validity evidence (Downing, 2003). This indicates that the assessment items measured the same abilities, establishing some commonalities between the constructs assessed in both examinations. The high correlation between student scores in both examinations confirms that the test items in both examinations measured the same construct/content areas derived from blueprints. The students obtained similar scores (and in seven instances, the same scores) on retesting as they received the first time. These data confirm that with the implementation of QA processes, it is possible to generate equivalent examinations that reproduce test scores with a high level of certainty (Downing \& Haladyna, 2009).

Consequential validity evidence relates to the impact of assessment on teaching and learning (Downing \& Haladyna, 1997). The reproducibility of the pass-fail decision is also a very important source of validity evidence (Downing, 2003; Downing \& Haladyna, 2009). The parallel examinations have reproduced assessment outcomes for students with a high level of certainty as both examinations identified the same poorly achieving student, who failed in both examinations and about two thirds (62\%) of the low achieving students in the first examination, also scored poorly in the second examination. Although there was an administrative error, the results of the par- allel examinations and the outcomes of the assessment on student scores have indicated no adverse consequences for the students. The examinations are equivalent in this sense. This suggests that, under the current assessment regime, the School should have confidence in the decision to allow students to "resit" the examination and achieve the "best score" based upon performance on either examination.

The QA processes developed by the School were initially focused on managing internal validity threats. The University typically manages external validity threats through Examination Office processes. On this occasion, the problem arose at the School/Examinations Office interface-a communication failure. A debriefing with staff identified further communications concerns, and subsequently an Accountability Matrix (Appendix 1) was developed to provide greater clarity about roles and responsibilities internally, and formalised relationships with the Examinations Office. There was also an opportunity to consider emergent sources of error; as a result training for the use of optical mark recognition (OMR) scanner was introduced for new staff and new software flags were developed to automatically check accuracy of data entry. Clearly potential sources of error and new threats for validity arise all the time, and QA processes need to be reviewed regularly.

The QA processes generate significant volume of data, but how much is needed for a moderate stakes examination? Schuwirth et al. (2011) suggest that three major inferences are required to quantify the consistency of an assessment instrument, as well as provide validity evidence for the observed scores. These inferences are: 1) would the students obtain the same score on the parallel test as they did on the actual test? 2) would the students take the same place in the rank ordering from best to worst performing student on the parallel test as they did on the actual test? 3) would the students obtain the same pass-fail decisions as they did on the actual test? The high positive correlation $(r=0.82)$ and the $67 \%$ precision accuracy of the observed variation between student scores in both examinations confirm that majority of the students have obtained similar scores (seven of them obtained exactly the same score in both examinations) in the second examination as they did in the first examination. Most of the students (65\%) have also taken the same place in the decile ranking order from best to worst performing student on both examinations. The students have also obtained the same pass/fail decisions, with the same student failing in both examinations. On this basis, there is strong evidence in support of the validity, reliability and equivalence of the two examinations and that no group of students has been advantaged in this process.

Caution should be taken in any generalisations drawn from this study. Different institutions have different policies and guidelines and QA processes for assessment which may affect the development of "equivalent" examinations for students. The group of students "resitting" the examination may not have been representative of the whole cohort which could make a difference to the outcomes of the study. Not all sources of error may have been identified and accounted for in this study, though we believe sufficient evidence has been marshaled in support of our conclusions about a medium stakes examination.

\section{Conclusion}

Medical educators need to devote more time to evaluating their assessment regimes to generate strong evidence of validity 
so that resulting data and grades are defensible. Statistical and correlational data in this case study support the view that welldefined QA processes reduce the threats to validity of assessment. The establishment of QA processes in the development of examinations can ensure content representativeness of the test materials, the reproducibility and generalisability of the scores, the statistical characteristics of the assessment questions and consistency of pass-fail decisions made from the assessment scores. Developing parallel examinations to address notions of fairness in university assessment policies is possible when strong QA is in place, and does not appear to advantage any group of students. The detection of errors and validity threats and revision of QA processes should be an ongoing activity.

\section{Acknowledgements}

The authors thank Dr Lisa Foa (the Unit Co-ordinator) and all the teaching staff who participated in developing the assessment items.

\section{REFERENCES}

Downing, S. M. (2002). Threats to the validity of locally developed multiple-choice tests in medical education: Construct-irrelevance variance and construct under-representation. Advances in Health Sciences Education, 7, 235-241. doi:10.1023/A:1021112514626

Downing, S. M. (2003). Validity: On the meaningful interpretation of assessment data. Medical Education, 37, 830-837. doi:10.1046/j.1365-2923.2003.01594.x

Downing, S. M. (2004). Reliability: On the reproducibility of assessment data. Medical Education, 38, 1006-1012. doi:10.1111/j.1365-2929.2004.01932.x

Downing, S. M., \& Haladyna, T. M. (1997). Test item development: Validity evidence from quality assurance processes. Applied Measurement in Education, 10, 61-82. doi:10.1207/s15324818ame1001 4

Downing, S. M., \& Haladyna, T. M. (2009). Validity and its threats. In S. M. Downing, \& R. Yudkowsky (Eds.), Assessment in health professions education (pp. 21-55). London: Routledge.

Fowell, S. L., Southgate, L. J., \& Bligh, J. G. (1999). Evaluating assessment: The missing link? Medical Education, 33, 276-281. doi:10.1046/j.1365-2923.1999.00405.x

Hamdy, H. (2006). Blueprinting for the assessment of health professsionals. The Clinical Teacher, 3, 175-179. doi:10.1111/j.1743-498X.2006.00101.x

Hays, R. (2008). Assessment in medical education: Roles for clinical medical educators. The Clinical Teacher, 5, 23-27. doi:10.1111/j.1743-498X.2007.00165.x

Jozefowicz, R. F., Koeppen, B. M., Case, S. M., Galbraith, R., Swanson, D. B., \& Glew, R. H. (2002). The quality of in-house medical school examinations. Academic Medicine, 77, 156-161. doi:10.1097/00001888-200202000-00016

Kane, M. (2006). Content-related validity evidence in test development. In S. M. Downing, \& T. M. Haladyna (Eds.), Handbook of test development (pp. 131-153). Mahwah, NJ: Lawrence Erlbaum Associates.

Malau-Aduli, B. S., Zimitat, C., \& Malau-Aduli, A. E. O. (2011). Quality assured assessment processes: Evaluating staff response to change. Journal of Higher Education Management \& Policy, 23, 1-23.

Malau-Aduli, B. S., \& Zimitat, C. (2011). Peer review improves the quality of MCQ examinations. Assessment \& Evaluation in Higher Education, 34, 1-13. doi:10.1080/02602938.2011.586991

Messick, S. (1989). Validity. In R. L. Linn (Ed.), Educational measurement (3rd ed., pp. 13-104). New York: American Council on Education and Macmillan.

Norcini, J., Anderson, B., Bollela, V., Burch, V., Costa, M. J., Duvivier, R., Galbraith, R., Hays, R., Kent, A., Perrott, V., \& Roberts, T. (2011). Criteria for good assessment: Consensus statement and recommendations from the Ottawa 2010 Conference. Medical Teacher, 33, 206-214. doi:10.3109/0142159X.2011.551559

Precht, D., Hazlett, C., Yip, S., \& Nicholls, J. (2003). Item analysis user's guide. Hong Kong: International Database for Enhanced Assessments and Learning (IDEALHK).

SAS (2009). Statistical Analysis System Institute, North Carolina USA v.9.2.

Schuwirth, L., Colliver, J., Gruppen, L., Kreiter, C., Mennin, S., Onishi, H., Pangaro, L., Ringsted, C., Swanson, D., Van der Vleuten, C. P. M., \& Wagner-Menghin, M. (2011). Research in assessment: Consensus statement and recommendations from Ottawa 2010 Conference. Medical Teacher, 33, 224-233. doi:10.3109/0142159X.2011.551558

Tavakol, M., \& Dennick, R. (2011). Post examination analysis of objective tests. Medical Teacher, 33, 447-458. doi:10.3109/0142159X.2011.564682 


\section{B. S. MALAU-ADULI ET AL.}

\section{Appendix}

Appendix 1.

Accountability Matrix for Written Examinations.

This matrix defines the tasks to be completed for the development of written exam papers in the MBBS (ordinary, deferred ordinary and supplementary exam papers). It denotes the person accountable for specific actions (1); those involved in the decision making processes (2) and those who will be informed of the outcomes of decisions (3).

\begin{tabular}{|c|c|c|c|}
\hline TASK & Unit Coordinator & MEU & PDA \\
\hline Assessment timelines and Excel workbook for entry of all assessment results & 2 & 1 & 3 \\
\hline Blueprints and weightings of questions for each assessment & 1 & 2 & 3 \\
\hline Initial Contacting of question writers & 1 & 2 & 3 \\
\hline Follow-up with question writers & 2 & 1 & 3 \\
\hline First draft of questions prepared & 2 & 1 & 3 \\
\hline Format Questions & 3 & 2 & 1 \\
\hline First draft of exam paper in KB & 3 & 1 & 3 \\
\hline First check of questions & 1 & 2 & 3 \\
\hline Peer review of questions & 1 & 2 & 3 \\
\hline Final draft of exam paper & 2 & 1 & 3 \\
\hline Format exam paper & 3 & 2 & 1 \\
\hline Final exam paper in KB for QA & 3 & 1 & 2 \\
\hline Sign-off on exam paper & 1 & 3 & 3 \\
\hline PDF copy of exam paper to Exams Office-InSite/print locally & 3 & 2 & 1 \\
\hline Shading of correct response on MCQ Answer Sheet & 2 & 2 & 1 \\
\hline Collection and collation of exam papers & 3 & 3 & 1 \\
\hline Scanning of MCQ answer sheets & 3 & 2 & 1 \\
\hline SAQs to examiners for marking & 2 & 3 & 1 \\
\hline Entry of students' results into excel workbook & 2 & 2 & 1 \\
\hline Sign-off on results & 1 & 2 & 3 \\
\hline Results to exams office & 2 & 3 & 1 \\
\hline Feedback to students \& staff & 2 & 1 & 3 \\
\hline
\end{tabular}

Note: 1 = Accountable; 2 = Decision making team; 3 = Information network; MEU = Medical Education Unit; PDA = Program delivery and assessment team.

1. Evaluation of assessment is a neglected area of academic practice.

2. The use of well-defined quality assurance (QA) processes in the development of assessment items/examinations in medical education contributes to the generation of data in support of validity arguments for assessment.

3. The use of QA processes contributes positively to giving confidence for the validity, reliability and fairness of parallel examinations in university settings.

4. New threats to validity of assessment arise continually, and need ongoing monitoring and management. 